M.G. Burton, R. Jayawardhana \& T.L. Bourke, eds.

\title{
ACS Coronagraphic Observations of Optically Thin Debris Disks
}

\author{
Mark Clampin \\ GSFC, 8077 Greenbelt Road, Greenbelt, MD 20771 \\ John Krist \\ STScI, 3700 San martin Drive, Baltimore, MD21218 \\ David R. Ardila, David A. Golimowski, Holland C. Ford \\ JHU, 34th and N. Charles Street, Baltimore, MD21218 \\ Garth Illingworth \\ UCO/Lick, University of California, Santa Cruz, CA 95064
}

\begin{abstract}
The Advanced Camera for Surveys (ACS) offers a coronagraphic imaging mode with angular resolution of $0.026^{\prime \prime} \mathrm{pixel}^{-1}$. In combination with with the appropriate subtraction of reference star point spread functions (PSF) the coronagraph is capable of achieving contrast ratios of $\sim 1000$. We present some of the first ACS observations of the optically thin debris disk HD141569A and discuss new results from these observations.
\end{abstract}

\section{Introduction}

The Advanced Camera for Surveys (ACS) is a third generation instrument for the Hubble Space Telescope's (HST) second decade of science operations. It was installed in HST during the fourth servicing mission (SM3B) in March 2002 and replaced the Faint Object Camera (FOC). ACS provides HST with a powerful complement of three new imagers; the Wide Field Camera (WFC), the High Resolution Camera (HRC) and the Solar Blind Camera (SBC). The HRC is a near-UV optimized, high resolution imager $\left(0.027^{\prime \prime}\right.$ pixel $\left.^{-1}\right)$, providing critically sampled images $(\lambda>500 \mathrm{~nm})$ over a $29^{\prime \prime} \times 26^{\prime \prime}$ field of view. It is equipped with a coronagraphic mode for high contrast imaging of the circumstellar environments of bright stars. The HRC coronagraphic occulters are located at a focal plane in the aberrated beam, resulting in relatively large $1.8^{\prime \prime}$ and $3.0^{\prime \prime}$ diameters masks. Krist et al. (2003) reported in detail initial performance results from the first ACS coronagraphic observations of bright stars. Figure 1 shows the azimuthally averaged radial surface brightness profiles for a direct F435W image of a star, the observed $\mathrm{F} 435 \mathrm{~W}$ profile when the star is centered on the small spot, and the observed radial profile when two sequential coronagraphic images are subtracted. When coronagraphic observations are carefully planned and com- 


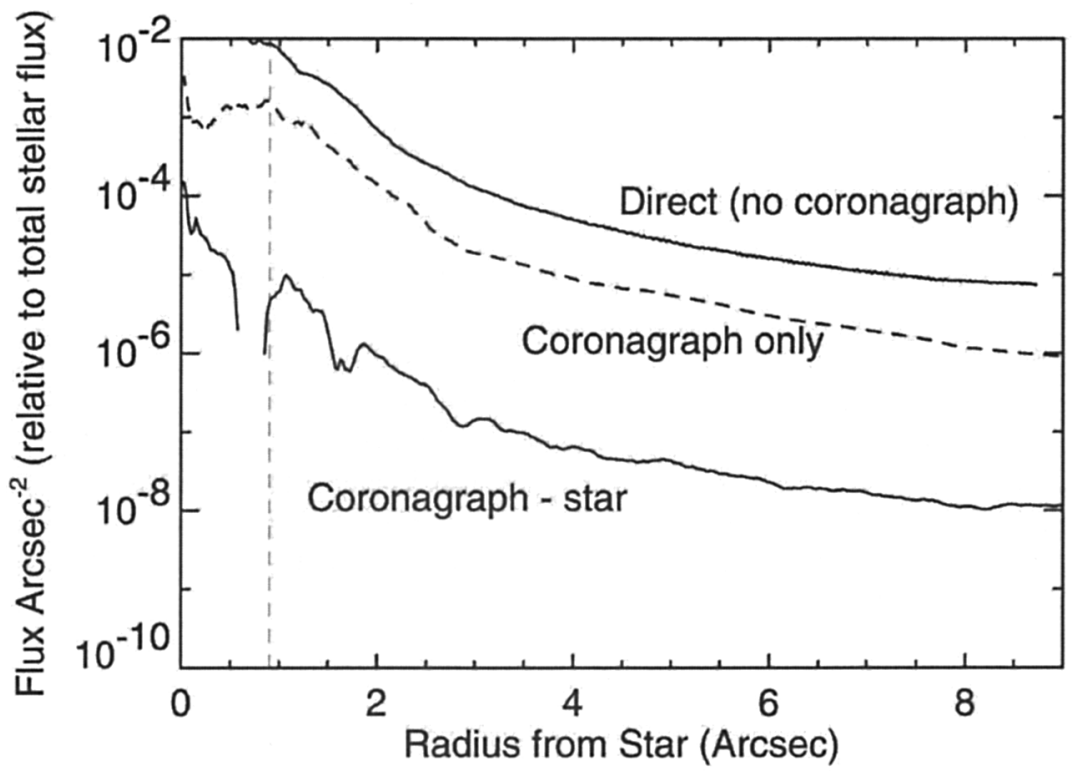

Figure 1. Demonstrated performance of the ACS coronagraph. The graph shows the effective contrast as a function of radial distance for the case of a coronagraphic observation (coronagraph only, and a coronagraphic observation with a reference star PSF subtracted (Coronagraph - Star). The HST PSF is shown for reference (Direct). 
parison stars of similar spectral type can be subtracted, to remove the residual wings of the central target star, it is possible to achieve contrast ratios of $\sim 1000$. The ACS Coronagraph is, therefore, a very capable instrument for studying the circumstellar environments of stars. The ACS Science Team has developed a program of coronagraphic imaging to conduct a survey of debris disks, primarily selected on the basis of their infrared excess. To date observations have been made of HD141569A, HD100546, HD162296 and GG Tau. In this contribution, we will focus on the new, multi-color observations of HD141569A, which fully demonstrate the scientific capabilities of the ACS Coronagraph.

HD 141569A (spectral type B9.5Ve; Jaschek \& Jaschek 1992) is one of a few stars with a detection of excess infrared radiation by IRAS, and for which an associated circumstellar debris disk has been imaged in reflected light. The first images of HD 141569A's circumstellar disk were obtained by NICMOS, and show complex structure (Weinberger et al. 1999, hereafter W99; Augereau et al. 1999) that might be attributed to the gravitational perturbation by one or more planets (W99; Mouillet et al. 2001, hereafter M01). Other studies conclude that the 5 Myr-old disk is too young to have formed Jovian planets (Weinberger et al. 2000) and have proposed alternative sculpting mechanisms, such as dust migration (Takeuchi \& Artymowicz 2001). The disk has a fractional infrared excess luminosity of $L_{\text {disk }} / L_{*}=8.4 \times 10^{-3}$ (Sylvester et al. 1996), and has been imaged to a radius of $\sim 500$ AU. Since HD 141569A's disk has an angular radius of $5^{\prime \prime}$ it is an ideal target for imaging with the ACS Coronagraph.

HD 141569A was observed with the HRC coronagraph on UT 2002 July 21 as part of the ACS Early Release Observation program (HST program 8992). A sequence of images was recorded over two orbits, after which $H S T$ was rolled by $28^{\circ}$ and the sequence was repeated. This roll offset permits the separation of PSF features, which are stationary with roll, from real disk structure, whose orientation on the detector changes with roll. Each observational sequence comprised exposures made with the broadband ACS filters F435W $(B)$, F606W (broad $V$ ), and F814W $(I)$. A reference star HD 129433 was observed immediately after HD 141569A with the same instrumental configuration. Although, both stars have the same spectral type, HD 129433 is less reddened $(B-V=-0.01$, versus 0.09 ). The images of HD 129433 were normalized to, aligned with, and subtracted from the corresponding images of HD 141569A. The normalization of HD 129433 with respect to HD 141569 is accurate to within $2 \%$, which means that the brightest regions of the disk have a photometric error of $\pm 0.02 \mathrm{mag}$. $\operatorname{arcsec}^{-2}$, while the fainter regions in the "gap" have photometric errors of \pm 0.1 mag. $\operatorname{arcsec}^{2}$.

\section{Results}

The PSF-subtracted images, shown in Figure 2, yield a more detailed view of the disk than seen in previous $H S T$ coronagraphic images obtained with NICMOS (W99; Augereau et al. 1999) and STIS (M01). The disk has four distinct annular zones: a clear zone within $\sim 175$ AU of the star, a bright "ring" with a sharp inner edge from $\sim 175 \mathrm{AU}$ to $\sim 215 \mathrm{AU}$, a faint zone from $\sim 215 \mathrm{AU}$ to $\sim 300 \mathrm{AU}$, and a broad "ring" from $\sim 300 \mathrm{AU}$ to $\sim 400 \mathrm{AU}$. (All distances are measured along the disk's southern semimajor axis.) The inner and outer 


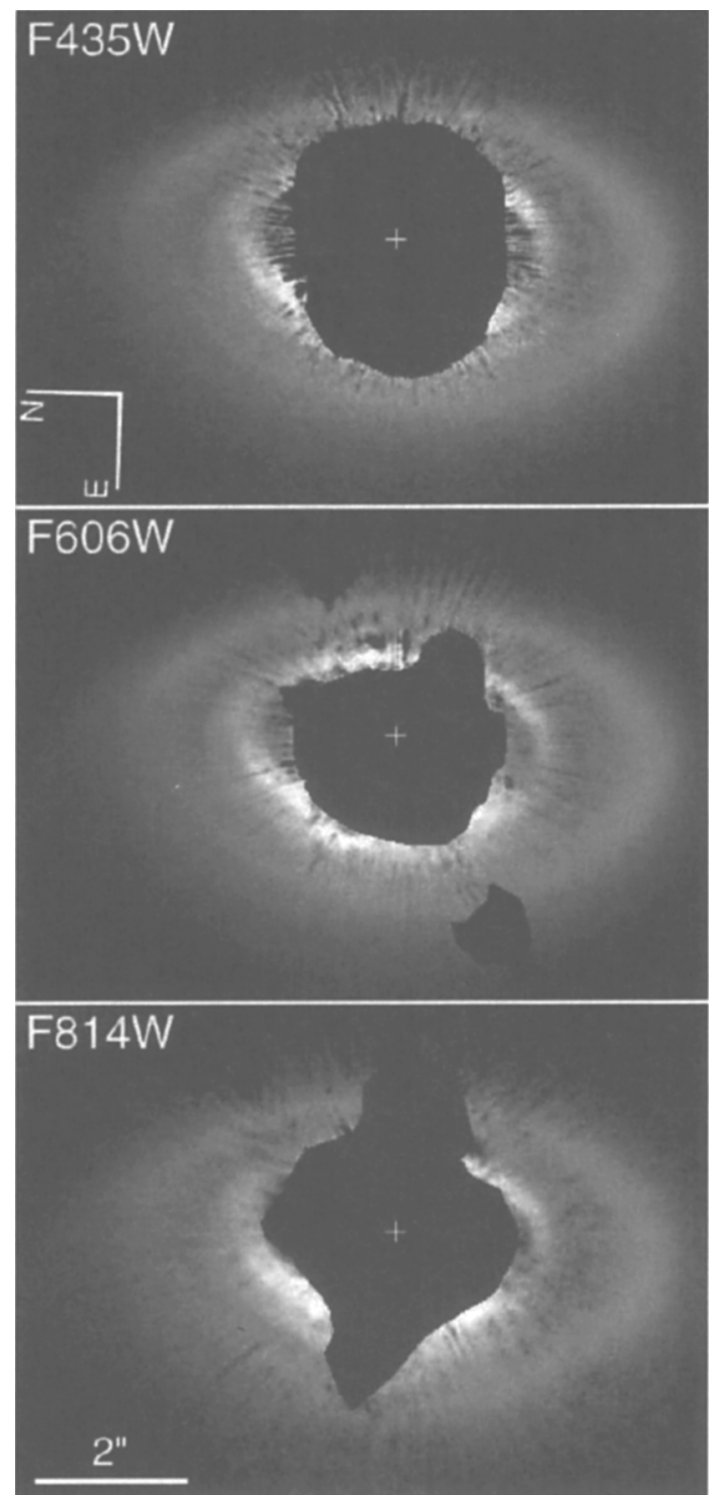

Figure 2. B (F435W), V (F606W), and I band images of HD 141569A's disk with the reference star PSF subtracted. The central region of each image has been masked to cover the occulting mask and related subtraction residuals. 
rings appear to be complex structures comprising thin and tightly wound spirals of dust. Moreover, the surface brightness of the outer ring varies azimuthally by a factor of 2.5. Faint, broad arcs of dust superposed upon fainter and more diffuse dust extend from the northeast and southwest regions of the disk. These arcs resemble open spiral arms. The diffuse dust in the northeast extends 1200 AU from HD 141569A. The southwest arc extends toward the binary star HD $141569 \mathrm{BC}$ located $\sim 8$. 5 to the northwest of HD 141569A.

Figure 3 shows the $V$-band surface brightness of the disk, derived from the combined $\mathrm{F} 606 \mathrm{~W}$ images after azimuthal filtering of the radial subtraction residuals. (The filtering technique is described below.) The north and east regions of the disk are generally brighter than the south and west regions. The F814W/F606W and F814W/F435W flux ratios of the disk are $10 \% \pm 0.7 \%$ and $25 \% \pm 2 \%$ larger, respectively, than those of HD $141569 \mathrm{~A}$, revealing that the disk is significantly redder than the star. These ratios are the same across the disk,indicating that its color is spatially uniform. Because the coronagraph's occulting spots lie in the aberrated beam from $H S T$, some unocculted, on-axis starlight reaches the HRC's corrective optics and is reimaged on the detector inside the image of the spot (Figure 1). This unocculted starlight allows direct measurement of the star's location in the image. Our images show that HD 141569A is not located at the geometric center of the circumstellar disk, but is offset by $25 \mathrm{AU}$ toward the west side. M01 found that the inner and outer disks are offset by $0.2 \mathrm{AU}$, however, our images clearly resolve the edge of the inner disk in the North-South direction and we find that the star is actually offset with respect to the disk.

The north-south orientation of the semimajor axis of the projected disk and the likely condition of forward scattering by dust indicate that the brighter, eastern side of the disk is nearer to us. Assuming that the disk is optically thin and flat (M01) and that the observed "rings" are circular, we coadded our F435W and F606W images and deprojected the resulting image to simulate a face-on view of the disk using an inclination angle of $51^{\circ}$ from face on (W99). The radial subtraction residuals seen in the constituent images were diminished by azimuthally filtering the combined image. Each pixel in the azimuthally filtered image was then multiplied by the square of its distance from HD 141569A to compensate for the radially diminishing stellar illumination. The enhanced brightness of the near side of the disk indicates strong forward scattering of starlight by the dust. W99 derived a scattering asymmetry parameter of $g=$ 0.11 (Henyey \& Greenstein 1941) for the disk based on a measured east/west brightness ratio of $1.5 \pm 0.2$ and an assumption of azimuthally uniform disk brightness. However, our images show that the disk is not azimuthally uniform. We fitted a Henyey-Greenstein phase function to our entire deprojected F435W + F606W image, accounting for the true inclination. We adjusted $g$ until the region of brightness enhancement along the disk's near side was made consistent with the overall azimuthal brightness trend. We obtained visually satisfactory results for $g=0.25-0.35$, which indicates more forwardly scattering dust than reported by W99. Figure 4 maps the product of the dust's albedo $(\omega)$ and its optical depth perpendicular to the plane of the disk $\left(\tau_{\perp}\right)$. This product is proportional to $\kappa_{s} \Sigma$, where $\kappa_{s}$ is the scattering cross section and $\Sigma$ is the surface density of the dust. 


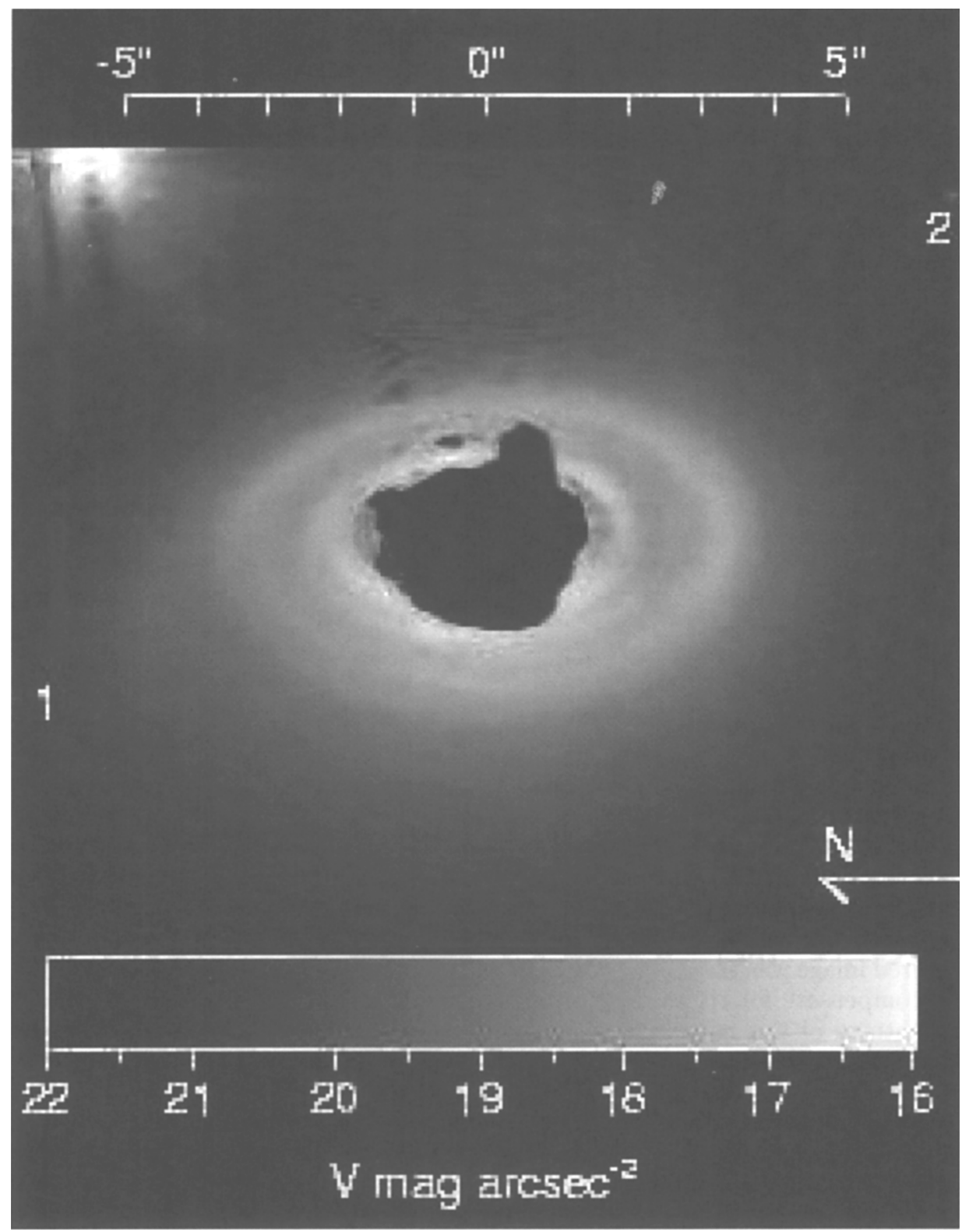

Figure 3. V-band surface brightness map of the HD141569A disk, obtained from the azimuthally filtered $\mathrm{F} 606 \mathrm{~W}$ image and photometrically calibrated from the synthetically derived flux of HD141569A in this band. HD141569BC lie in the upper right corner, and two background stars are labelled 1 and 2 . 


\section{Discussion}

The diverse spiral structure of HD 141569A's disk prompts us to reconsider the potential influence of a low-mass companion on the disk's morphology. W99 have suggested that the depleted region of the disk from $\sim 215-300$ AU could be produced from tidal clearing by a Jupiter-sized planet on the gap itself. Both Takeuchi \& Artymowicz (2001)and Klahr and Lin (2002)have argued that sharp structures in disk may not always be indicators of embedded planets. Current theories suggest that planet formation at such large distances from the star is unlikely within the 5 Myr age of this system (Boss 1998), and Takeuchi \& Artymowicz (2001) have argued that the creation of the circular depleted region by an outwardly migrating planet is unlikely. However, the presence of HD141569BC may induce scattering from inner orbits to the gap, and Thommes (2002) have shown that planets within the inner $100 \mathrm{AU}$ may scatter objects to the $300 \mathrm{AU}$ region. Again, these mechanisms have to be able to circularize the orbit of the planet within 5 Myrs. On the other hand, a perturbing object responsible for the gap does not need to be in the gap itself: an inner object might clear the gap by excitation of density waves within its outer Lindblad resonance (Goldreich \& Tremaine 1978). These possibilities remain to be investigated, and at this point we cannot exclude a planet as the mechanism responsible for the observed structures.

The open and extended spiral arms, the apparent association of the southwest arm with HD 141569BC, the large azimuthal density variations of the disk, and the $\sim 25$ AU offset of HD 141569A relative to the center of the disk are characteristics consistent with the tidal effects of encounters between circumstellar disks and passing stars (Pfalzner, Henning, \& Kley 2000; Larwood \& Kalas 2001). If HD 141569A and HD 141569BC are bound, then a few hundred encounters between the disk and HD 141569BC could have occurred over 5 Myr. Under such circumstances, the disk would be tidally truncated at a distance of $\sim 0.2-0.4$ times the semimajor axis of the orbit and its outer regions would develop spiral structure (Artymowicz \& Lubow 1994). The eccentricity of the HD 141569A/BC system would also induce eccentricity in the disk that could account for the displacement of the disk with respect to HD 141569A. Detailed dynamical modelling of the system is currently underway to explore the interaction of the disk with HD 141569BC. The causes of the complicated structures observed in the dust disks around HD 141569A and other young stars clearly require further investigation and modeling (Ardila et al. in preparation). As our images demonstrate, the ACS coronagraph can produce disk imaging with unprecedented resolution and contrast. Such high-quality images permit the investigations necessary to address quantitatively the many outstanding questions regarding the composition, dynamics, and evolution of infrared excess dust disks.

\section{References}

Artymowicz, P., \& Lubow, S. H. 1994, ApJ, 421, 651

Augereau, J., Lagrange, A., Mouillet, D., \& Ménard, F. 1999, A\&A, 350, L51

Henyey, L. G., \& Greenstein, J. L. 1941, ApJ, 93, 70 


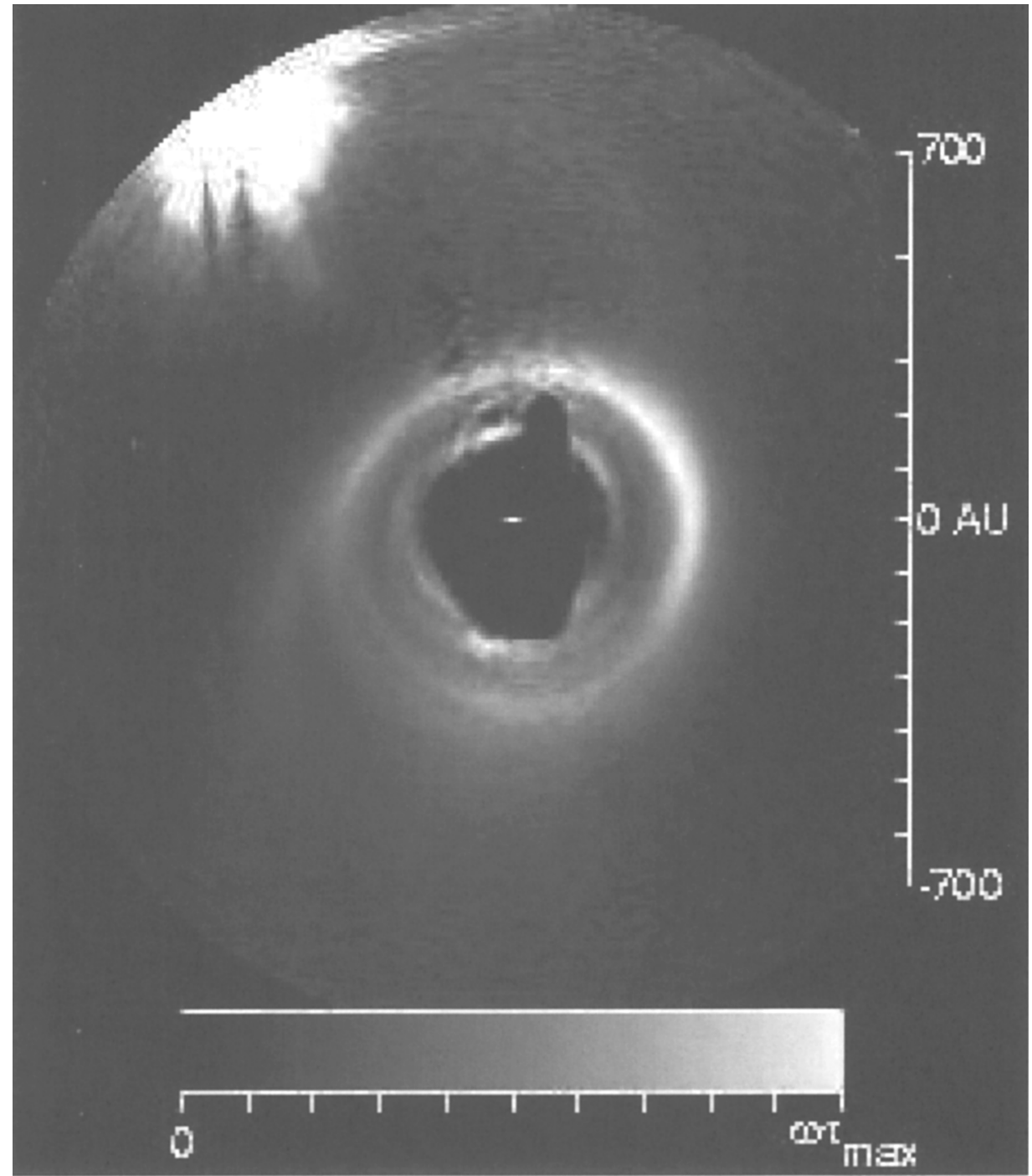

Figure 4. Greyscale map of the disk's apparent optical depth (i.e. the product of the dust albedo $\omega$ and vertical depth $\tau$ ). The line of sight is towards the botton and north is towards the left. 
Jaschek, C., \& Jaschek, M. 1992, A\&AS, 95, 535

Krist, J. E., Hartig, G. F., Clampin, M., Golimowski, D. A., Ford, H. C., \& Illingworth, G. D. 2003, Proc. SPIE, vol. 4860 (in press).

Klahr, H. H., \& Lin, D. N. C. 2000, ApJ, 554, 1095

Larwood, J. D., \& Kalas, P. G. 2001, MNRAS, 323, 402

Mouillet, D., Lagrange, A., Augereau, J., \& Ménard, F. 2001, A\&A, 372, L61

Pfalzner, S., Henning, T., \& Kley, W. 2000, in Poster Papers, IAU Symp. 200, Birth and Evolution of Binary Stars, ed. B. Reipurth \& H. Zinnecker, 193

Sylvester, R. J., Skinner, C. J., Barlow, M. J., Mannings, V. 1996, MNRAS, 279,915

Takeuchi, T., \& Artymowicz, P. 2001, ApJ, 557, 990

Thommes E.W., \& Duncan, M.J. \& Levison, H.F. 2002, AJ, 123, 2862

Weinberger, A. J., Becklin, E. E., Schneider, G., Smith, B. A., Lowrance, P. J., Silverstone, M. D., Zuckerman, B., \& Terrile, R. J. 1999, ApJ, 525, L53

Weinberger, A. J., Rich, R. M., Becklin, E. E., Zuckerman, B., \& Matthews, K. 2000, ApJ, 544, 937 